\title{
Reporte preliminar del sismo del 13 de abril de 2007, Guerrero, México
}

\author{
Sara Ivonne Franco*, Caridad Cárdenas-Monroy, Aída Quezada-Reyes, Carlos Valdés-González, \\ Casiano Jiménez-Cruz , Luis Edgar Rodríguez-Abreu y Javier Ortiz-Castro \\ Contribuciones hechas por: (en orden alfabético) \\ Arturo Cárdenas-Ramírez, José Luis Cruz-Cervantes, Jorge Alberto Estrada-Castillo, \\ Ángeles Gutiérrez-García, Iván Rodríguez-Rasilla, Bernardino Rubí-Zavala, Yi Tan Li y \\ Alfredo Yañez-Soto
}

Servicio Sismológico Nacional, Instituto de Geofísica, Universidad Nacional Autónoma de México,

México, D.F., 04510.

*ivonne@ssn.ssn.unam.mx

\section{Resumen}

El 13 de abril del 2007 a las 05:42 hrs. (tiempo GMT) se registró, en 27 estaciones de la Red sismológica de Banda ancha, un evento de magnitud Mw = 5.8 proveniente de la costa de Guerrero, México, muy cerca de la ciudad de Acapulco. El mecanismo focal corresponde a un mecanismo de fallamiento inverso con un plano de falla casi vertical. El epicentro de este evento se localiza en la denominada "Brecha de Guerrero", sin embargo, la profundidad a la que se ubica (aproximadamente 30 km) no muestra una correlación directa con la zona sismogénica y por tanto no es posible asociar el evento a una relajación de esfuerzos en esta región.

La aceleración máxima observada es de alrededor de 90 gales y se registró en la estación CAIG localizada a menos de 20 km de distancia del epicentro. El análisis de atenuación de las aceleraciones indican un sismo más energético que lo que se había observado para sismos mexicanos de estas características.

Palabras clave: Brecha de Guerrero, atenuación, mecanismos focales, sismos intraplaca.

\section{Abstract}

On april 13, 2007 at 05:42 (Universal Time) an earthquake, with magnitude Mw=5.8, occurred in the state of Guerrero, Mexico, near the Pacific Coast. The event was recorded in 27 stations that comprise the Broadband Seismic Network, managed by the National Seismological Service of Mexico.

The source parameters show an interplate thrust earthquake with an almost vertical fault plane. The epicentral location lies within the Guerrero seismic gap. Nevertheless, the hypocentral depth of $30 \mathrm{~km}$ makes it difficult to associate this earthquake to a possible stress relaxation in this gap.

The largest peak ground acceleration was recorded at CAIG, a broad-band station located less than $20 \mathrm{~km}$ from the epicenter. The attenuation curve suggests a very energetic earthquake in comparison with other Mexican interplate events.

Key words: Guerrero gap, attenuation, focal mechanism, interplate earthquake. 


\section{Introducción}

El 13 de abril de 2007, a las 05:42 horas (00:42, tiempo del centro de México), se registró un evento de magnitud $\mathrm{Mw}=5.8\left(\mathrm{M}_{\mathrm{E}}=6.3\right)$, cerca del Puerto de Acapulco. El epicentro con coordenadas $17.168^{\circ} \mathrm{N}, 100.379^{\circ} \mathrm{W}$, se localizó en la zona de subducción de la costa del Pacífico mexicano. La réplica principal tuvo una magnitud de $\mathrm{Mw}=5.3\left(\mathrm{M}_{\mathrm{E}}=5.4\right)$ y se registró aproximadamente tres horas después del evento principal. Otra réplica importante ocurrió a las 04:26 horas del 13 de abril, la magnitud fue $\mathrm{M}_{\mathrm{E}}=4.9$.

La costa del Pacífico mexicano es una zona de gran actividad sísmica. Esta actividad es consecuencia de la interacción entre la placa continental de Norte América y las placas oceánicas de Cocos y Rivera (Figura 1). Los eventos que se registraron el día 13 de abril de 2007 se localizan en la zona denominada "Brecha sísmica de Guerrero", a mayor profundidad que la zona sismogénica, lo que los hace especialmente interesantes.

La aceleración máxima que se registró en las estaciones de la red de Banda Ancha del Servicio Sismológico Nacional (SSN) fue de aproximadamente 90 gales y se registró en la estación de CAIG, localizada en el poblado de Cayaco, Guerrero aproximadamente a $50 \mathrm{~km}$ al noreste del Puerto de Acapulco y a $20 \mathrm{~km}$ del epicentro (Figura 2).

En este reporte presentamos un análisis preliminar de los parámetros y características principales del sismo del 13 de abril de 2007. Todos los resultados que mostramos son obtenidos a partir de los datos del SSN.

\section{Marco Tectónico}

Los límites de placas tectónicas concentran la mayor actividad sísmica del mundo. En México interactúan cinco placas: Norte América, Caribe, Cocos, Pacífico y Rivera (Figura 1). La subducción de la placa de Cocos bajo la placa de Norte América ha generado más de 70 sismos de magnitud M > 6.5 en el siglo pasado (Kostoglodov y Pacheco, 1999). El principal rasgo tectónico de esta zona de convergencia es la Trinchera Mesoamericana, la cual se extiende paralelamente a la costa desde Jalisco-Colima hasta Centroamérica, a lo largo de $3000 \mathrm{~km}$. El sismo de Michoacán del 19 de septiembre de $1985(\mathrm{M}=8.1)$, localizado a más de $400 \mathrm{~km}$ de la Ciudad de México, que causó la muerte de más de 6000 personas y ocasionó numerosos daños materiales, es sólo un ejemplo del peligro sísmico que representa esta zona para algunas ciudades importantes del país.

La zona de subducción de la placa de Cocos bajo la placa de Norteamérica abarca el segmento de costa de $\operatorname{los} 15^{\circ}$ a $20^{\circ}$ de latitud Norte, y de $92^{\circ}$ a $105^{\circ}$ de longitud Oeste (Burbach et al., 1984). El ángulo de subducción de la placa de Cocos con respecto a la placa de Norteamérica, en la porción comprendida entre Michoacán y Oaxaca, es de aproximadamente $12^{\circ}$ lo cual esta considerado como un ángulo de subducción bajo. La velocidad de convergencia entre estas placas aumenta hacia el sudeste, variando de 4.8 $\mathrm{cm} / \mathrm{año}$, en $104^{\circ} \mathrm{W}$, a $7.5 \mathrm{~cm} / \mathrm{año}$, en $94^{\circ} \mathrm{W}$ (Pardo y Suárez, 1995, DeMets et al., 1994).

La zona central de Guerrero, inmediatamente al sur de la zona de ruptura de los temblores de Petatlán (1979) y Michoacán (1985), localizada entre los paralelos $16.9^{\circ}$ y $17.2^{\circ} \mathrm{N}$ y los meridianos $100.1^{\circ}$ y $101.0^{\circ} \mathrm{W}$ (Figura 1 ), se considera que es una brecha sísmica con un alto potencial sísmico. De acuerdo con el catálogo de Anderson et al. (1989) el evento más reciente que ha ocurrido en esta zona es en 1911, con una magnitud aproximada de 7.5. A esta región se le denomina Brecha sísmica de Guerrero, y pertenece a las 5 brechas maduras que se habían identificado en 1981 (Singh et al., 1981).

Actualmente se considera que la Brecha sísmica de Guerrero tiene un potencial sísmico capaz de producir un terremoto de magnitud $\mathrm{M}_{\mathrm{w}}=8.1$ - 8.4 (Suárez et al., 1990), en caso de que el área total de la brecha rompiera en un solo evento (cabe mencionar que este escenario no se ha observado en el pasado reciente, por lo menos 500 años), lo que significa un peligro potencial para ciudades como México y Acapulco entre otras.

\section{Evento principal}

El evento ocurrido el 13 de abril del 2007 se registró en un total de 27 estaciones de la Red de Banda Ancha del SSN. Cada una de estas estaciones se encuentra equipada con un digitizador de 24 bits Quanterra-Q330, un sismómetro triaxial STS-2, el cual permite registrar ondas sísmicas con respuesta plana a la velocidad del suelo en una banda de frecuencias entre 0.01 a $30 \mathrm{~Hz}$, un reloj GPS, así como un acelerómetro triaxial Episensor que registra las aceleraciones del suelo dentro de un amplio espectro de frecuencias sin saturación de la señal para sismos grandes locales y regionales.

El evento se localizó en la costa de Guerrero, con epicentro en las coordenadas $17.168^{\circ} \mathrm{N}, 100.379^{\circ} \mathrm{W}$ y a una profundidad de $32.7 \mathrm{~km}$. Dentro de la región epicentral se cuenta con 4 estaciones de Banda ancha (ZIIG, CAIG, MEIG y PLIG; Figura 2).

A partir de mediciones geodésicas, Franco (2006) determinó que el área acoplada va desde los $10 \mathrm{~km}$ de profundidad hasta aproximadamente los $30 \mathrm{~km}$ y se extiende una distancia de hasta $100 \mathrm{~km}$ a partir de la trinchera en dirección del buzamiento. Como se puede ver en la vista de perfil de la Figura 2, este evento se localiza justo en el límite de la zona sismogénica y coincide con el doblamiento de la placa.

\section{Solución de tensor de Momentos}

Actualmente el SSN está en proceso de implementar el cálculo automático del tensor de momento sísmico (CAT- 

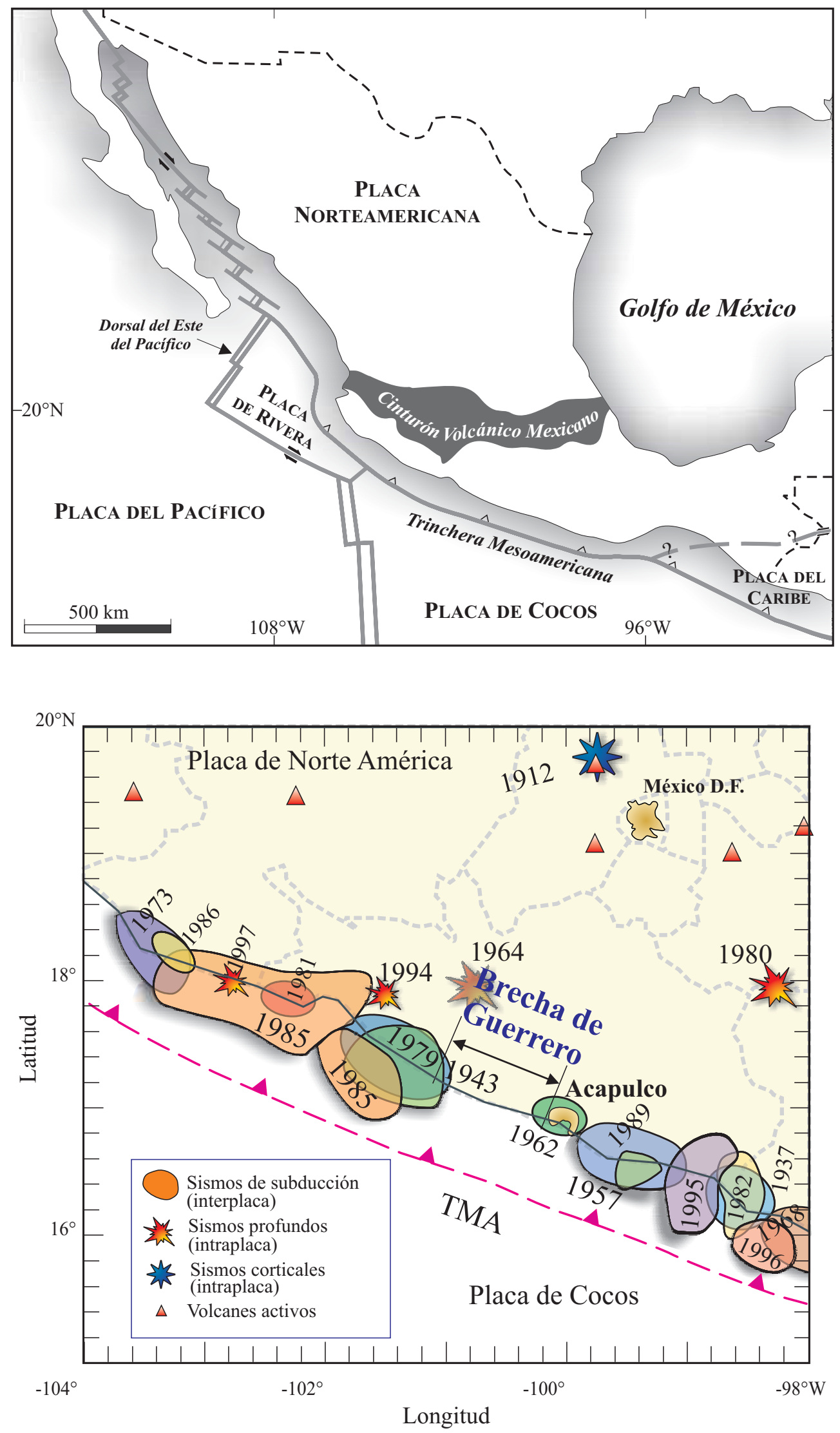

Figura 1. Izquierda: Mapa de tectónico de México, se muestran las placas que interactúan en el territorio, así como sus límites. Derecha: Sismotectónica del área de estudio, las áreas sombreadas representan las zonas de ruptura para los eventos de magnitud M>7.0 que ocurrieron durante el siglo pasado. Se muestra la ubicación geográfica de la Brecha de Guerrero (modificada a partir de Lowry et al., 2001 

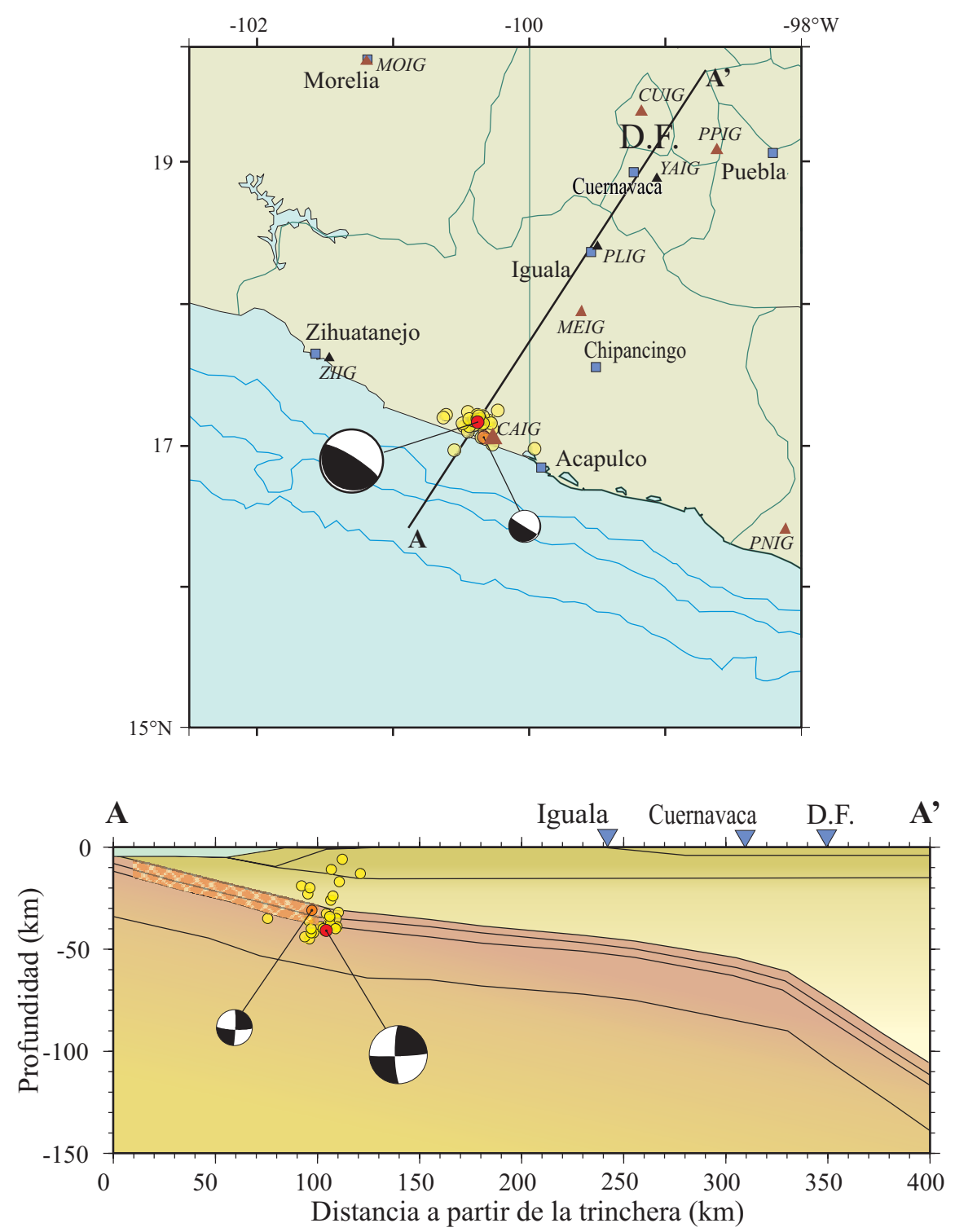

Figura 2. Distribución epicentral del evento principal del 13 de abril de 2007 (mecanismo focal mayor), así como las réplicas asociadas (círculos de color amarillo), incluyendo la de mayor magnitud ( $\mathrm{MW}=5.3$ ). Arriba: Localización en planta del evento principal y las réplicas. También se muestra la localización de las estaciones más cercanas al epicentro (triángulos), así como las principales ciudades (cuadrados). Abajo: Vista en sección (perfil A-A') de la localización del evento del sismo principal y sus réplicas. La geometría de la placa es tomada de Cárdenas-Monroy (2003). La parte sombreada indica la zona acoplada determinada por Franco (2006).

MS) a partir de la inversión de la forma de onda (Franco et al., 2002; Nolasco-Carteño, 2006). Para este evento fue posible obtener el tensor de momentos de manera casi automática. La información que se obtuvo a partir del tensor permitió tener una visión preliminar de la importancia sismotectónica del evento, así como determinar el momento sísmico, el cual es el parámetro escalar de la fuente puntual del doble par. Este parámetro está relacionado con el tamaño y el desplazamiento promedio de la falla (Aki, 1966), y a través de éste se obtiene la magnitud de momento $\left(\mathrm{M}_{\mathrm{w}}\right)$, la cual no presenta límites de saturación. El CATMS requiere la localización precisa del evento, ya que el resultado es sensible a la localización.
El tensor de momento sísmico calculado por el SSN se obtuvo durante la primera hora después de ocurrido el evento. El mecanismo generador del temblor sugiere un fallamiento casi vertical (Figura 3).

Al comparar el resultado obtenido a partir del CATMS y la solución generada a través del cálculo estándar del "Quick Centroid Moment Tensor" (CMT) del proyecto Global del CMT (antes de la Universidad de Harvard) (http://www. globalcmt.org/CMTsearch.html), la cual se publicó aproximadamente 8 horas después de ocurrido el evento, podemos constatar que la solución que generó el SSN es confiable e inmediata. Lo mismo ocurre al comparar la solución del SSN con la del "NaTional Earthquake Information Center" 
Tangencial
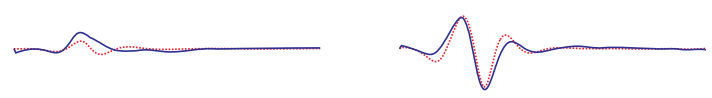

MEIG f0.05.data $\mathrm{HH}, 44 \mathrm{Max} A m p=5.59 \mathrm{e}-02 \mathrm{~cm}$ $\mathrm{VR}=7 \overline{9} .4$
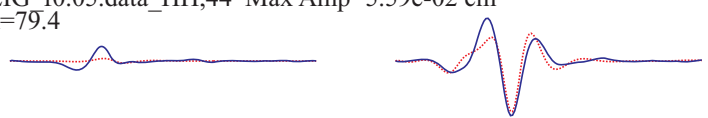

PLIG f0.05.data_HH,34 Max Amp=3.42e-02 cm $\mathrm{VR}=81.4$

YAIG f0.05.data_HH,36 Max Amp=2.70e- $02 \mathrm{~cm}$ $\mathrm{VR}=77.5$
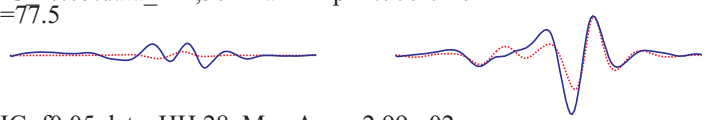

CUIG f0.05.data_HH,28 Max Amp=2.99e-02 cm $\mathrm{VR}=75.6$
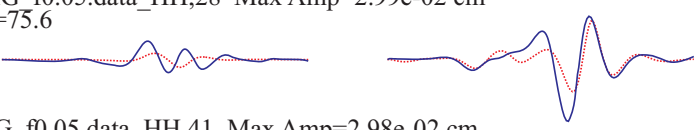

PPIG f0.05.data_HH,41 Max Amp=2.98e-02 cm $\mathrm{VR}=67.2$

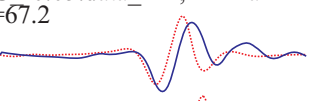

MOIG f0.05.data_HH_3.43 Max Amp=2.30e-02 cm

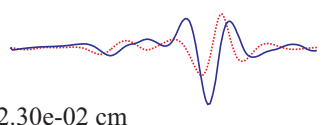
$\mathrm{VR}=2.9$
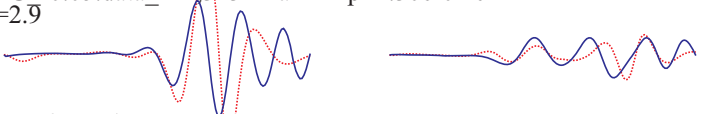
PLIG f0.05.data_HH,31 Max Amp $=5.38 \mathrm{e}-03 \mathrm{~cm}$
VR=84.0

YAIG f0.05.data_HH,34 Max Amp=4.36e- $03 \mathrm{~cm}$ $\mathrm{VR}=8 \overline{3} .9$

CUIG f0.05.data_HH,26 Max Amp=4.32e-03 cm $\mathrm{VR}=6 \overline{9} .1$

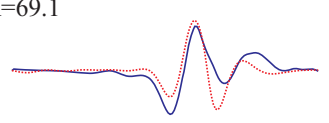

MOIG f0.05.data_HH,343 Max Amp=2.36e-03 cm

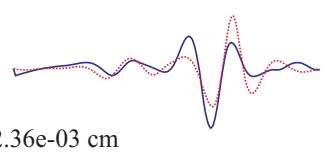
$\mathrm{VR}=62.1$
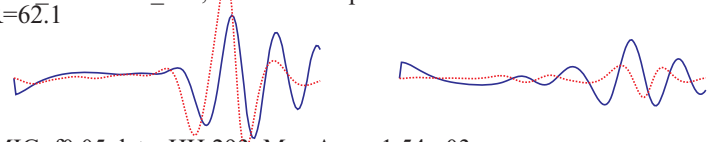

MMIG f0.05.data_HH,293 Max Amp $=1.54 \mathrm{e}-03 \mathrm{~cm}$ $\mathrm{VR}=-17.3$
MMIG f0.05.data_HH,29: Max Amp $=8.36 \mathrm{e}-03 \mathrm{~cm}$ $\mathrm{VR}=-107.0$

Tangencial

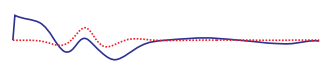

$\underset{\mathrm{VRIG}}{\mathrm{M}=55.0} \mathrm{f} 0$
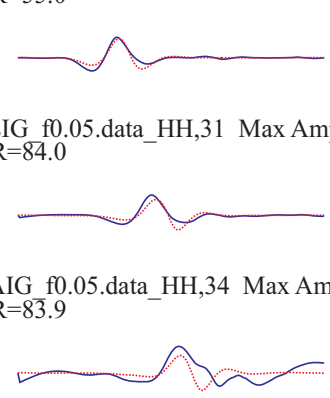

Radial
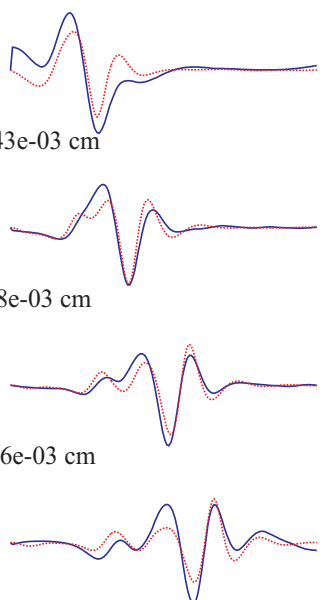

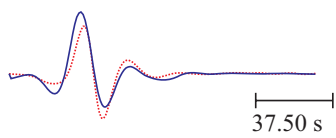

Strike $=302 ; 122$

Rake $=90 ; 91$

Dip $=79 ; 11$

Mo $=5.48 \mathrm{e}+24$

$\mathrm{Mw}=5.8$

Porcentaje DC $=86$

Porcentaje CLVD $=14$

Porcentaje ISO $=0$

Varianza $=1.52 \mathrm{e}-05$

Var. Red $=6.24 \mathrm{e}+01$

RES/Pdc. $=1.77 \mathrm{e}-07$
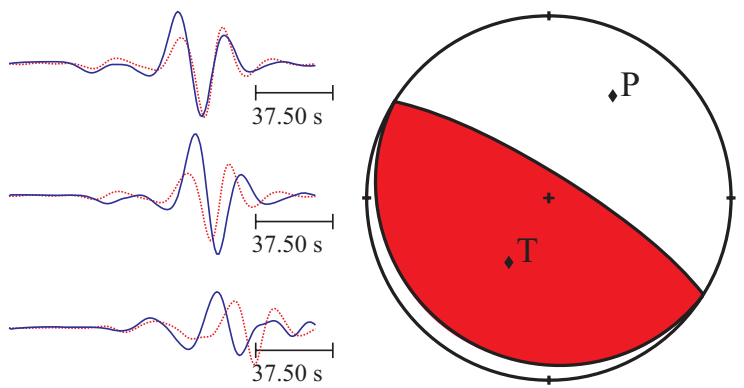

Figura 3. Solución del tensor de momentos a partir de la inversión de la forma de onda, se utilizaron 7 estaciones del SSN para obtener este resultado. Arriba: Solución para el evento principal. Abajo: Solución para la réplica de mayor magnitud. La línea puntead indica la forma de onda sintética mientras que la línea continua es para los datos. 
Tabla 1. Parámetros de la fuente del sismo del 13 de abril del 2007, en Guerrero, México.

\begin{tabular}{lcccccccc}
\hline Entidad & Lat $^{\circ}$ & Long $^{\circ}$ & $\begin{array}{c}\text { Profundidad } \\
(\mathrm{km})\end{array}$ & $\begin{array}{c}\text { Mo } \\
(\mathrm{dyn} \cdot \mathrm{cm})\end{array}$ & Strike Dip & Rake \\
\hline $\mathrm{CMT}^{(\mathrm{a})}$ & 17.41 & -100.21 & 38.9 & $9.9 \times 10^{24}$ & 284 & 73 & 91 \\
NEIC $^{(\mathrm{b})}$ & 17.398 & -100.099 & 33 & $1.3 \times 10^{25}$ & 291 & 79 & 98 \\
Local $^{(\mathrm{c})}$ & 17.168 & -100.379 & 32.7 & $5.5 \times 10^{24}$ & 302 & 79 & 90 \\
\hline
\end{tabular}

a. Solución preliminar del CMT de Harvard (http://www.globalcmt.org/ CMTsearch.html). b. Solución preliminar del CMT de NEIC < <http://neic. usgs.gov/neis/FM/>>. c. Solución preliminar del CMT a partir de datos regionales $(\mathrm{SSN})$.

(NEIC) (http://neic.usgs.gov/neis/FM/). La Tabla 1 resume los parámetros del evento del 13 de abril de 2007 obtenidos por las diferentes instituciones.

El mecanismo focal del evento principal muestra un fallamiento inverso, mientras que, como se puede observar en la parte inferior de la Figura 3, el fallamiento de la réplica principal esta representado por un mecanismo de falla normal. Sin embargo, los planos de falla de ambos eventos son casi verticales y los ejes de presión y tensión en ambos mecanismos resultan casi paralelos, tal como se puede observar en la Figura 2.

\section{Movimientos fuertes}

La determinación del valor de aceleración máxima es de gran importancia para la estimación del movimiento de estructuras construidas por el hombre. Se han realizado múltiples esfuerzos para determinar una estimación teórica del valor de la aceleración máxima que nos ayude a mitigar los efectos destructivos de un gran evento (por ejemplo el trabajo de García et al., 2005). Es en este sentido que realizamos el siguiente análisis de las aceleraciones máximas producidas durante este sismo.

El movimiento fuerte del terreno se mide a través de la aceleración del suelo que se registra en los observatorios sismológicos.

En este trabajo los valores de aceleración máxima se obtuvieron derivando los registros de velocidad de las estaciones sismológicas de banda ancha del SSN para cada una de las componentes. En la Tabla 2 se muestran dichos valores para cada estación y para cada componente.

Como se discutirá adelante, para comparar los valores de aceleración máxima producidos por este evento con una curva teórica promedio representativa de sismos mexicanos intraplaca (García et al., 2005) hemos considerado utilizar como aceleración máxima $\left(\mathrm{A}_{\max }\right)$ la media cuadrática de las aceleraciones pico de las componentes horizontales, esto es:

$$
A_{\max }=\sqrt{\frac{A_{N}^{2}+A_{E}^{2}}{2}}
$$

Donde $A_{N}$ es el valor de aceleración pico en la componente norte-sur y $A_{E}$ es el valor de la aceleración pico en la componente este-oeste.

La distancia $R$ se ha considerado como la distancia hipocentral.

La Figura 4 muestra la distribución de la aceleración máxima con respecto a la distancia para las estaciones que registraron el evento.

Para obtener una curva de atenuación de las aceleraciones se llevó a cabo una regresión ponderada de los datos de aceleración de este sismo. La ponderación se consideró como el inverso de la distancia entre la estación y el epicentro, de tal manera que las estaciones más cercanas son más pesadas que aquéllas que se encuentran más alejadas de la fuente.

La ecuación de la curva que mejor ajusta nuestros datos es la siguiente:

$$
\log A_{\max }=3.263-\log R-0.0023 R
$$

Donde $A_{\max }$ es la aceleración máxima horizontal en gales (la que se estimó a partir de la ecuación 1) y $R$ es la distancia hipocentral, al evento, dada en $\mathrm{km}$. El valor del coeficiente del término $\log R$ se fijo a 1 (García et al., 2005).

La expresión propuesta por García et al., (2005) no considera estaciones localizadas a una distancia $R$ mayor a los $600 \mathrm{~km}$. Para comparar la curva de atenuación del evento del 13 de abril con la curva teórica obtenida a partir de la expresión de García et al., (2005) sólo hemos utilizado el registro de las estaciones que cumplan con la distancia máxima para la que fue desarrollada dicha expresión (700 $\mathrm{km}$ ), después de esta distancia la curva pierde resolución.

En un radio menor a los $100 \mathrm{~km}$ de distancia epicentral la cobertura de estaciones es baja, ya que sólo está la estación CAIG, localizada en el poblado de Cayaco en el estado de Guerrero, México. Dicha estación se ubica a 18.7 km de distancia del epicentro. Esta estación registró el mayor valor de Amax, que fue de 92.02 gales. La siguiente estación más cercana es MEIG ubicada en el mismo estado en el poblado de Mezcala, a $119 \mathrm{~km}$ del epicentro y registró una aceleración máxima de 26.03 gales. La calidad del registro en ambas estaciones es buena y no hay saturación de la señal, lo que permite tener confianza en las observaciones.

La Figura 4 muestra la curva que mejor se ajusta a las observaciones de aceleración máxima. Además, se ha incluido la curva teórica obtenida a partir de la expresión propuesta por García et al. (2005) para sismos mexicanos intraplaca y considerando una magnitud $\mathrm{M}_{\mathrm{w}}=5.8 \mathrm{y}$ una profundidades de $32.7 \mathrm{~km}$. El decaimiento de la aceleración observada para distancias cercanas a la fuente queda representado por el decaimiento teórico, es decir, en un rango de distancias de 0 a $170 \mathrm{~km}$ todas nuestras observaciones quedan dentro del margen de incertidumbre de la curva teórica. La estación MEIG, localizada a una distancia aproximada de $120 \mathrm{~km}$ del epicentro, muestra un comportamiento anómalo en la 
Tabla 2. Aceleraciones máximas, en gales, del sismo del 13 de abril obtenidas para cada componente a partir de los registros de velocidad de las estaciones de banda ancha del SSN.

\begin{tabular}{|c|c|c|c|c|c|c|}
\hline Estación & Ubicación & $\begin{array}{l}\text { Distancia hipocentral } \\
\qquad(\mathrm{km})\end{array}$ & $\begin{array}{c}\mathrm{Az} \\
\text { (gales) }\end{array}$ & $\begin{array}{c}\text { An } \\
\text { (gales) }\end{array}$ & $\begin{array}{c}\mathrm{Ae} \\
\text { (gales) }\end{array}$ & $\begin{array}{l}\text { A max } \\
\text { (gales) }\end{array}$ \\
\hline ANIG & Auacatlán, Nayarit & 628.2 & 0.008 & 0.162 & 0.15 & 0.156 \\
\hline GAIG & Cayaco, Guerrero & 18.7 & 82.059 & 97.502 & 86.198 & 92.024 \\
\hline CCIG & Comitán, Chiapas & 920.7 & 0.067 & 0.074 & 0.13 & 0.106 \\
\hline CGIG & Casas Grandes, Chih & 1691.7 & 0.0415 & 0.039 & 0.026 & 0.033 \\
\hline CJIG & Chamela, Jalisco & 579.1 & 0.218 & 0.276 & 0.224 & 0.251 \\
\hline CMIG & Matias Romero, Oax & 611.1 & 0.235 & 0.109 & 0.26 & 0.199 \\
\hline CUIG & Ciudad Universitaria & 274.7 & 2.338 & 3.455 & 3.546 & 3.501 \\
\hline DHIG & Demacú, Hidalgo & 378.5 & 0.355 & 0.683 & 0.49 & 0.594 \\
\hline HPIG & Hidalgo del Parral, Chih & 1234.7 & 0.031 & 0.037 & 0.036 & 0.037 \\
\hline HUIG & Huatulco, Oaxaca & 499.1 & 0.006 & 0.0001 & 0.236 & 0.167 \\
\hline LPIG & La Paz, Baja California Sur & 1345.7 & 0.003 & 0.003 & 0.003 & 0.003 \\
\hline LVIG & Laguna Verde, Veracruz & 523.4 & 0.073 & 0.056 & 0.066 & 0.061 \\
\hline MEIG & Mezcala. Guerrero & 119.5 & 16.727 & 19.957 & 17.478 & 18.758 \\
\hline MMIH & Maruata, Michoacán & 352.9 & 0.451 & 0.93 & 0.851 & 0.893 \\
\hline MOIG & Morelia, Michoacán & 293.3 & 2.031 & 5.173 & 3.969 & 4.610 \\
\hline OXIG & Oaxaca, Oaxaca & 405.6 & 0.653 & 1.01 & 0.852 & 0.934 \\
\hline PLIG & Iguala, Guerrero & 167.3 & 4.52 & 4.119 & 4.739 & 4.440 \\
\hline PNIG & Pinotepa Nacional, Oaxaca & 264.4 & 0.973 & 0.585 & 1.099 & 0.880 \\
\hline PPIG & Volcán Popocatépetl & 287.1 & 2.317 & 6.28 & 4.982 & 5.668 \\
\hline SCIG & Sabancuy, Quintana Roo & 1040.5 & 0.009 & 0.249 & 0.225 & 0.237 \\
\hline TPIG & Tehuacan, Puebla & 363.1 & 1.117 & 1.423 & 2.043 & 1.761 \\
\hline TUIG & Tuzandépetl, Veracruz & 669.0 & 0.009 & 0.249 & 0.25 & 0.237 \\
\hline YAIG & Yautepec, Morelos & 237.7 & 2.132 & 4.594 & 6.042 & 5.367 \\
\hline ZAIG & Zacatecas, Zacatecas & 668.4 & 0.067 & 0.068 & 0.084 & 0.076 \\
\hline ZIIG & Zihuatanejo, Guerrero & 130.8 & 2.133 & 4.595 & 5.941 & 5.311 \\
\hline
\end{tabular}

atenuación al presentar valores muy altos y fuera de rango en el valor Amax.

Por otro lado, para las estaciones que se localizan a una distancia epicentral mayor de los $170 \mathrm{~km}$, los valores de aceleración son generalmente más altos que los obtenidos teóricamente. Esto podría significar que el sismo en estudio ha sido más energético que lo proyectado por la curva teórica para sismos intraplaca, lo cual podría deberse al inusual mecanismo de ruptura así como a la zona en la cual se localiza.

El SSN reportó la magnitud del evento como $\mathrm{M}_{\mathrm{E}}=6.3$. Sin embargo, la magnitud de momento sísmico es $\mathrm{M}_{\mathrm{w}}=5.8$ (ver Tabla 1 para más detalles). Dado que el cálculo de la magnitud $M_{E}$ se basa en la estimación de la energía sísmica radiada registrada en la estación CUIG, localizada en Cd. Universitaria, (Singh y Pacheco, 1994) y, como se discutió anteriormente, este evento ha sido más energético que lo que se había observado y además muestra un mecanismo de falla inusual en esta zona, es posible que en el trabajo de Singh y Pacheco (1994) no se hayan utilizado sismos que tuvieran patrones de radiación similares a este, siendo ésta una posible razón de la diferencia entre ambas magnitudes.

En la Figura 5 se muestra el mapa de isoaceleración del sismo principal del 13 de abril. Este mapa se generó interpolando las aceleraciones obtenidas a partir de 25 estaciones de la Red de Banda ancha. Los valores graficados son los que corresponden a la aceleración horizontal Amax.

Los valores mayores de aceleración se registraron en la zona más cercana al epicentro, donde la aceleración excedió los 90 gales (Figura 5).

La curva de isoaceleración de 60 gales coincide aproximadamente con la región donde se localizaron los epicentros de las réplicas del sismo principal.

Se puede observar que las aceleraciones decaen más rápido con la distancia, en dirección al estado de Oaxaca (dirección sureste) que en dirección al estado de Michoacán. Además, dado el efecto de sitio de la estación PPIG, ubicada en el volcán Popocatépetl, las aceleraciones registradas son más altas que las registradas en la estación YAIG, Morelos, estación que se ubica más cerca del epicentro.

\section{Mapa de Isosistas}

Para generar el mapa de isosistas del sismo del día 13 de abril de 2007 que se muestra en la Figura 6, se utilizó el 


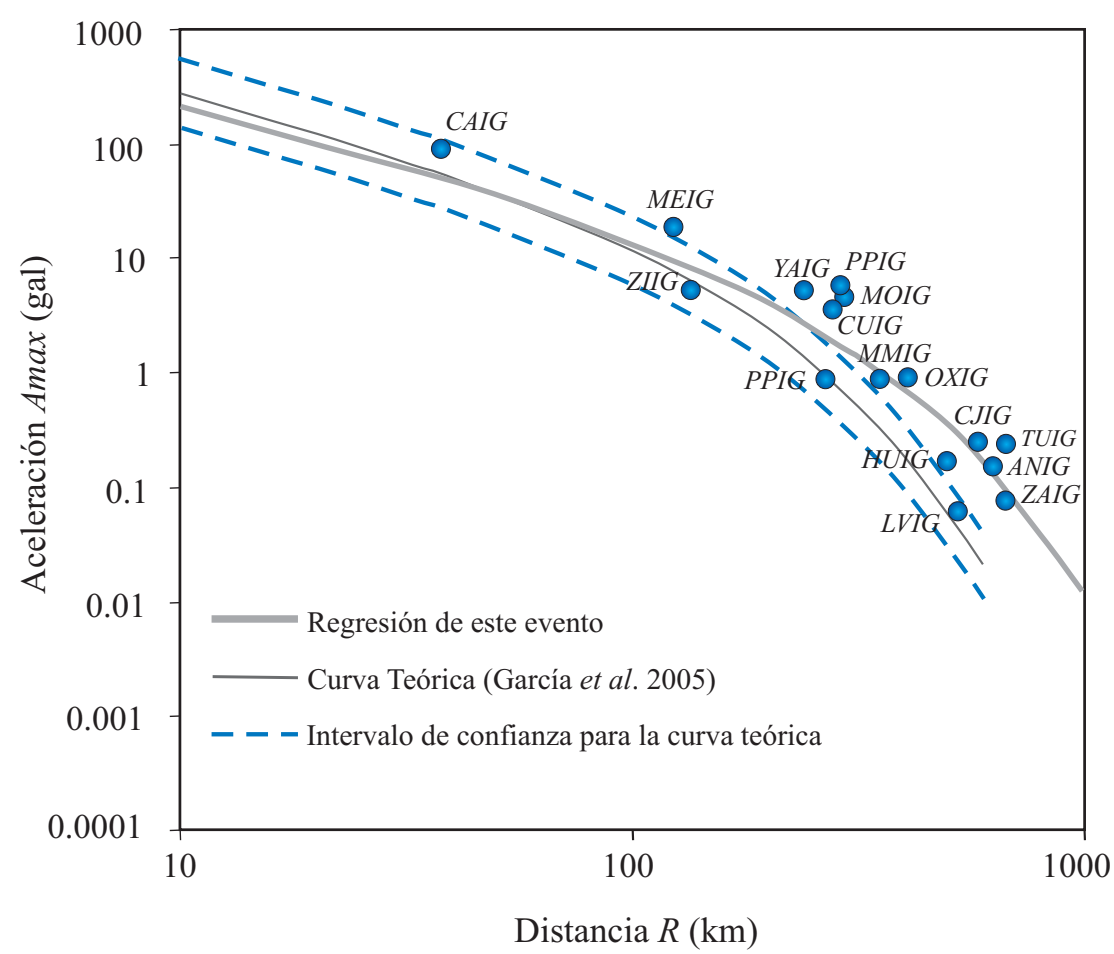

Figura 4. Aceleración pico horizontal Amax contra distancia hipocentral. Con línea continua gruesa se muestra la curva obtenida a partir de la regresión de los datos obtenidos para este sismo. Con línea continua delgada se muestra la curva teórica propuesta por García et al. (2005), y sus rangos de confiabilidad se muestran con líneas punteadas.

método de interpolación de Kriging, el cual consiste en un conjunto de rutinas de regresión lineal donde el parámetro a interpolar puede ser tratado como una variable regional.

Los datos que se utilizaron para la interpolación se obtuvieron a partir de reportes enviados por el público de manera voluntaria al portal de Internet del SSN (www. ssn.unam.mx/SSN/reporta-sismo.html). Dichos reportes permiten clasificar la intensidad del evento en términos de observaciones y comportamientos humanos así como en la respuesta del suelo y estructuras al movimiento. Para determinar la intensidad se toma como referencia la escala de Mercalli modificada. El valor de intensidad que se reporta por estado es un promedio de los valores reportados por municipio (Tan Y. y Jiménez-Cruz, 2002).

Para este sismo se recibieron un total de 592 reportes procedentes de los estados de Guerrero, Colima, Jalisco, Michoacán, Morelos, Puebla, Veracruz, Estado de México y Distrito Federal. El número de reportes de cada localidad, así como el valor de intensidad promedio, en la escala de Mercalli, se muestran en la Tabla 3.

Los límites de las isosistas fueron ajustados también a partir de la interpolación de los valores promedio de algunos municipios.

En la Figura 7 se muestra el mapa de isosistas del sismo del 9 de diciembre de 1965 (Figueroa, 1974), de magnitud 6.8. La localización de este evento $\left(16.55^{\circ} \mathrm{N}, 100.15^{\circ} \mathrm{W}\right.$ y profundidad de $35 \mathrm{~km}$ ) es similar a la reportada para el sismo del 13 de abril de $2007\left(17.168^{\circ} \mathrm{N}, 100.379^{\circ} \mathrm{W}\right.$ y profundidad de $32.7 \mathrm{~km}$ ). Comparando ambos mapas (Figuras 6 y 7) se observa que las curvas de intensidades son semejantes. Sin embargo, el área que corresponde a la isosista con valor de intensidad V para el sismo de 1965 $(\mathrm{M}=6.8)$ es menor que la registrada para el sismo de 2007 $\left(\mathrm{M}_{\mathrm{w}}=5.8\right)$. Esto puede atribuirse al incremento de población y construcciones en los diferentes estados durante los últimos años.

A pesar de que el reporte de intensidades puede ser muy subjetivo, al comparar el mapa de isosistas del sismo del 13 de abril (Figura 6) con el mapa de isoaceleraciones (Figura 5) se observa que los lugares donde se registraron mayores aceleraciones coinciden con los lugares donde se reportaron los mayores valores de intensidad sísmica.

\section{Procesado y difusión de la información}

Para obtener la magnitud del sismo del 13 de abril del presente año, se utilizó el programa de cómputo "PICA", desarrollado por técnicos del SSN. Con este programa se obtienen dos magnitudes sísmicas, $\mathrm{M}_{\mathrm{A}}$ y $\mathrm{M}_{\mathrm{E}}$ a partir del registro de la estación de banda ancha CUIG que se localiza en la Facultad de Veterinaria en Ciudad Universitaria, México, D.F. El cálculo de la magnitud $\mathrm{M}_{\mathrm{E}}$ consiste en la estimación de la energía sísmica radiada mientras que la magnitud $\mathrm{M}_{\mathrm{A}}$ se basa en la amplitud de las trazas de velocidad filtradas con un paso de banda de 15 a 30 segundos (Singh y Pache- 


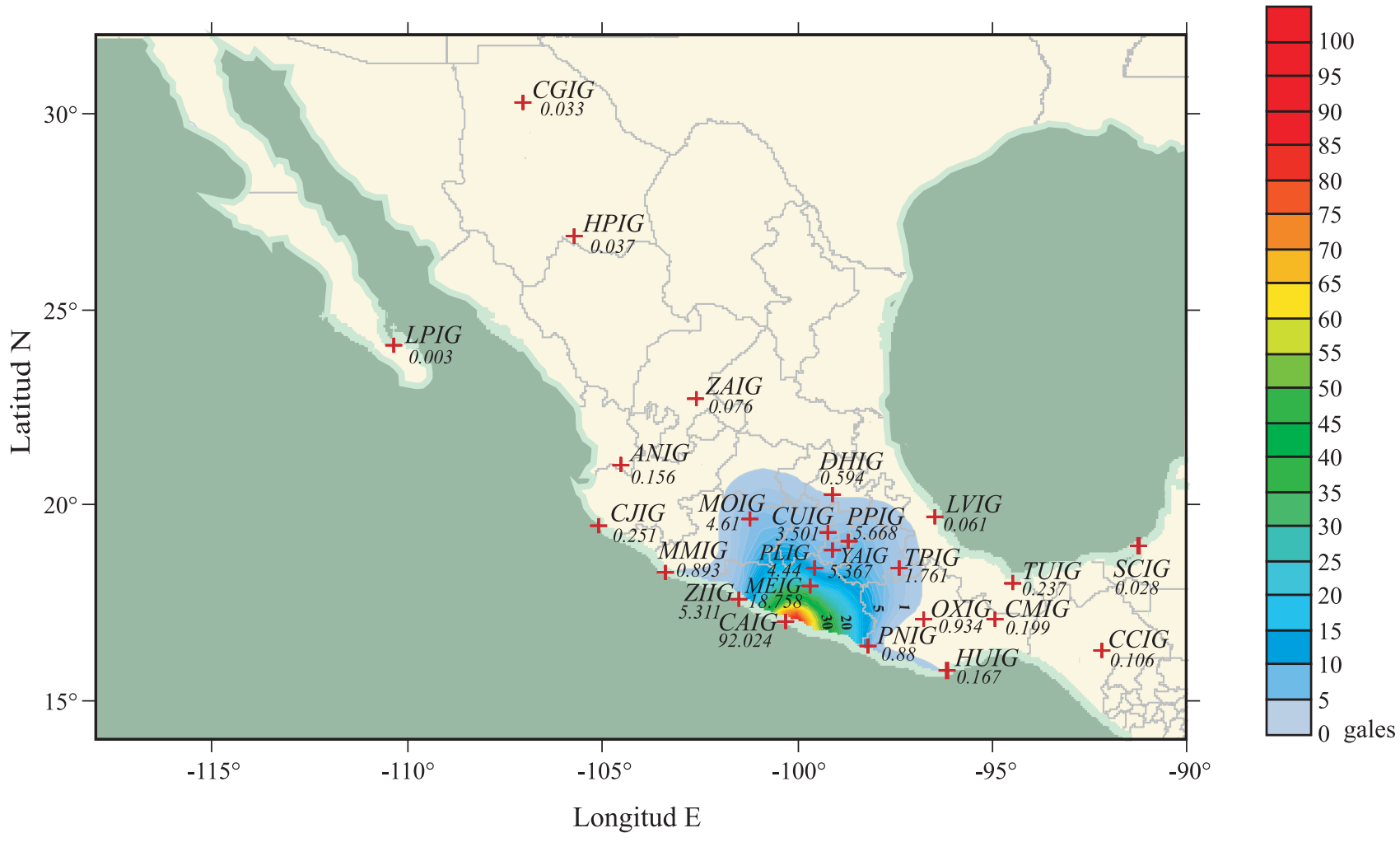

Figura 5. Mapa de isoaceleraciones. Las cruces indican las estaciones del SSN que registraron el evento y el número la aceleración máxima medida en cada una de ellas. El color blanco muestra aceleraciones menores a 1.0 gal.

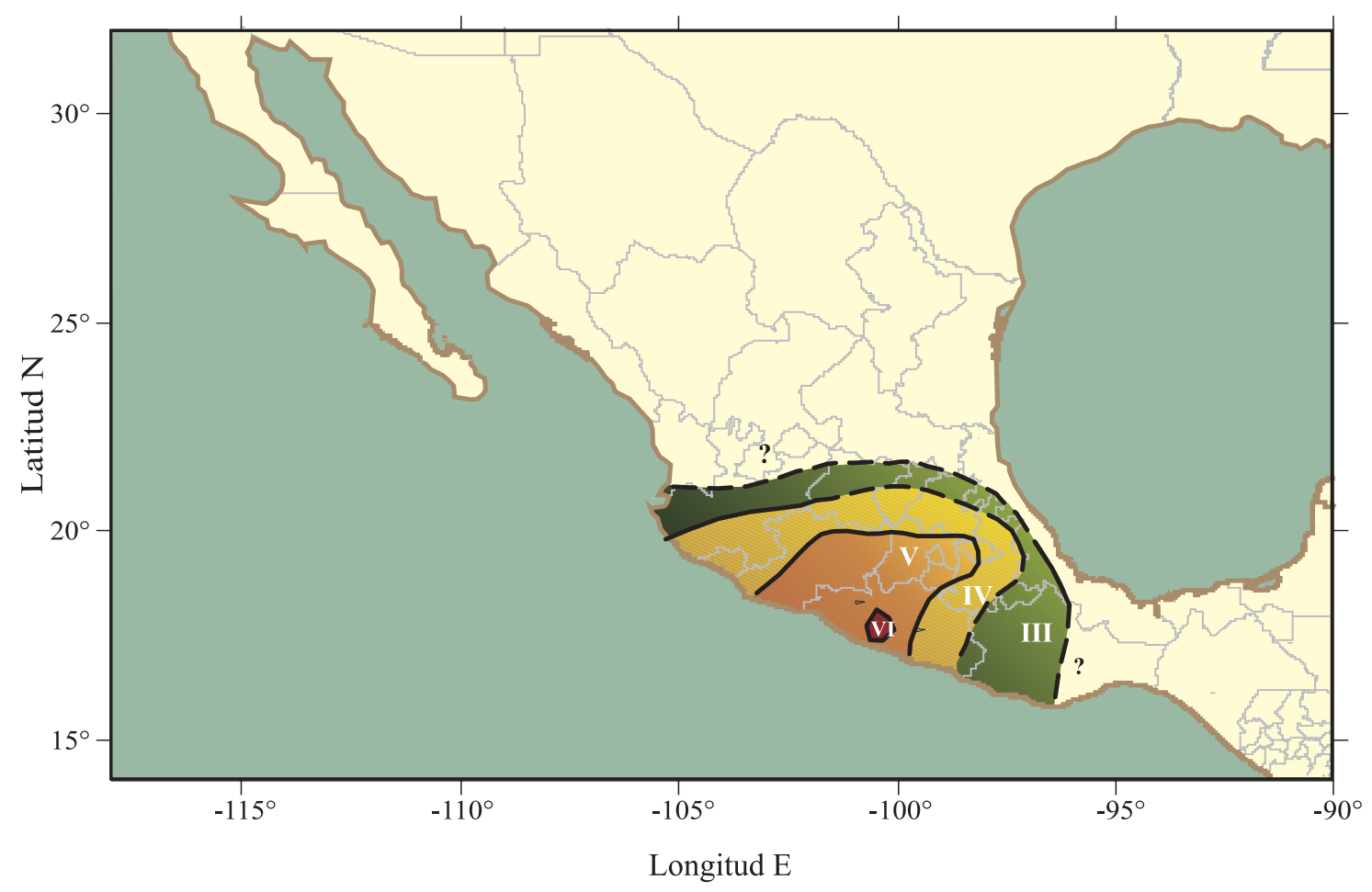

Figura 6. Mapa de isosistas. Las intensidades se tomaron a partir de los reportes enviados al portal de Internet del SSN. Las zonas de mayor intensidad corresponden a las zonas de mayor aceleración. Para más detalle ver el texto. 
Tabla 3. Intensidades reportadas al SSN por el público.

\begin{tabular}{lcccc}
\hline Estado & Intensidad & Promedio & $\begin{array}{c}\text { Número de } \\
\text { reportes }\end{array}$ & $\begin{array}{c}\text { Municipios } \\
\text { con reportes }\end{array}$ \\
\hline Colima & III-V & IV & 8 & 3 \\
Distrito Federal & III-X & V & 301 & 15 \\
Guerrero & III-X & V & 73 & 12 \\
Jalisco & III-VI & IV & 8 & 4 \\
Edo. de México & III-X & V & 95 & 21 \\
Michoacán & III-VI & V & 21 & 8 \\
Morelos & III-VIII & V & 32 & 8 \\
Puebla & III-VII & V & 44 & 6 \\
Veracruz & III-VI & IV & 10 & 5 \\
\hline
\end{tabular}

co, 1994). La distancia del epicentro a la estación CUIG, calculada también por este programa, permite obtener una primera estimación de la región donde ocurrió el evento. Sin embargo, para determinar la ubicación precisa del epicentro, es necesario contar con los registros procedentes de un mínimo de tres estaciones.

En los primeros minutos después de haber ocurrido el sismo, fue posible tener información preliminar sobre la hora exacta de ocurrencia del evento, región epicentral y magnitud. Esta información fue distribuida en todo momento vía telefónica y a través de la actualización automática del portal de Internet del SSN (www.ssn.unam.mx), lo cual fue posible utilizando programas desarrollados por el personal de este Servicio. Este procedimiento permite la disponibilidad de dicha información al público en muy poco tiempo.

Los parámetros principales del evento (región epicentral, profundidad y magnitud) fueron determinados utilizando los registros digitales de un total de 27 observatorios sismológicos pertenecientes a la Red de Banda Ancha, la Red del Valle de México y la Red Convencional, todas estas redes pertenecientes al SSN. Las trazas de las formas de onda (registros) se transmiten en tiempo real a la estación central ubicada en el Instituto de Geofísica de la Universidad Nacional Autónoma de México.

La información acerca de las características del evento fue enviada de forma inmediata vía fax y correo electrónico a instituciones gubernamentales, privadas y educativas, tales como Protección Civil: Distrito Federal, Estado de México, Acapulco, Hidalgo; Secretaría de la Defensa Nacional, Secretaría de Transportes y Comunicaciones, Comisión Federal de Electricidad, Telmex, Sistema Estatal de Protección, CENAPRED, SAT, TV Azteca, Televisa, Prensa Latina, Notimex, Cruz Roja, Universidad Autónoma de Puebla, UNAM, entre otros.

La pronta respuesta del SSN a la demanda de información, en el caso concreto de las dependencias de Protección Civil, facilita la toma de decisiones y la ejecución de medidas necesarias para canalizar ayuda en caso de que ésta sea requerida.

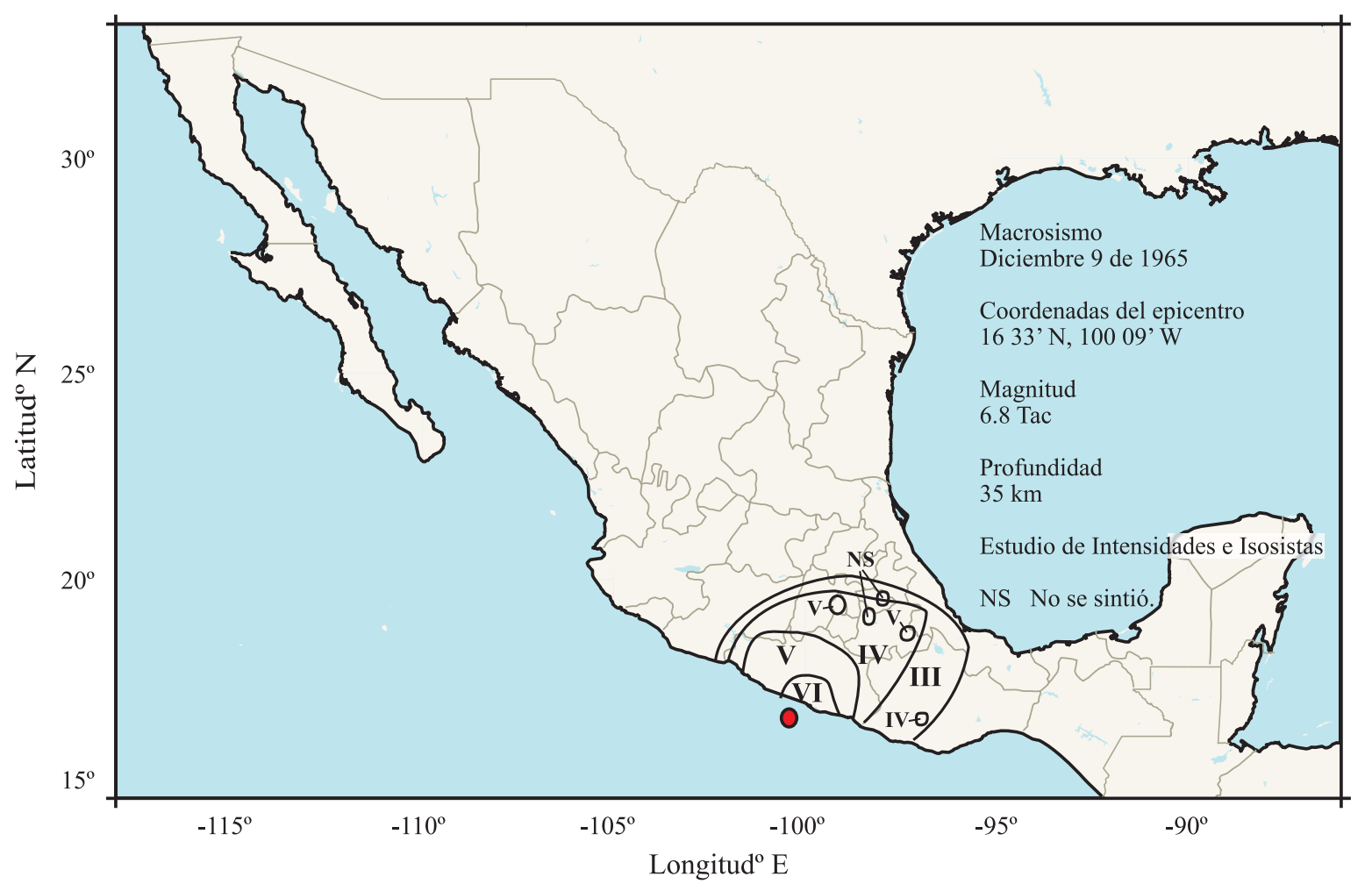

Figura 7. Mapa de isosistas del sismo del 9 de diciembre de 1965, de magnitud 6.8 (Tomado de Figueroa, 1974). 


\section{Conclusiones}

El análisis preliminar del evento del día 13 de abril de 2007 a partir de los datos obtenidos por el SSN nos permite determinar que se trata de un sismo con características particulares: Su ubicación geográfica, ya que se localiza en la misma región de la Brecha Sísmica de Guerrero y en el límite de la zona acoplada, lo convierte en un evento de gran interés para la comunidad sismológica.

El mecanismo focal del sismo es representativo de un fallamiento inverso, con un plano de falla casi vertical y poco común en eventos intraplaca. La réplica principal presenta un mecanismo de tipo normal, con un plano de falla casi vertical, muy parecido al que se observa con el evento principal. Esta observación invita a realizar análisis más detallados del estado de esfuerzos de la placa, así como de la historia de liberación de esfuerzos para este sismo en particular.

Los valores de las aceleraciones máximas, obtenidos a partir de los registros de velocidad de las estaciones que comprenden la Red de Banda Ancha, para las estaciones ubicadas a una distancia mayor a $170 \mathrm{~km}$ del epicentro y cuyos valores son más altos que los propuestos en análisis anteriores, probablemente estén relacionados con la naturaleza energética del evento, misma que podría relacionarse con el mecanismo de ruptura, el patrón de radiación y la propagación de las ondas sísmicas.

Se recomienda llevar a cabo un estudio acerca de la interacción de esfuerzos de este sismo con las zonas circundantes. Así mismo, un realizar un análisis detallado de las réplicas de este evento permitirá proponer un modelo de fuente y determinar la geometría de la falla, con lo cual se podrá definir la historia de ruptura de la fuente. La combinación de esta información con datos de deformación obtenidos a partir de mediciones geodésicas permitiría tener una visión más clara acerca del estado de esfuerzo en la Brecha de Guerrero.

La constante expansión de la Red de Banda Ancha del SSN, la modernización de sus equipos, técnicas de transmisión y procesamiento de la información en los últimos años permite disponer de una mayor cantidad de registros sísmicos de gran calidad, lo cual se traduce en resultados más precisos en el área de investigación, así como en mejorar la capacidad de respuesta ante la ocurrencia de sismos en el territorio nacional.

\section{Agradecimientos}

Agradecemos al técnico del Servicio Sismológico Nacional. José Antonio Santiago. El proyecto de expansión del SSN ha sido posible gracias al fideicomiso 2038 FONDEN - Alertamiento sismológico de la Secretaría de Gobernación. Agradecemos la colaboración de Alejandro Hurtado durante este proceso de expansión. Agradecemos a Enedina Martínez y al personal administrativo del SSN.
Los autores agradecemos a David Escobedo, Carles Canet y Daniel Gracía-Jiménez por sus comentarios. Así mismo, agradecemos al Dr. Marco Guzmán-Speziale y al árbitro anónimo cuyos comentarios han servido para mejorar el presente trabajo.

\section{Referencias bibliográficas}

Aki, K., 1966, Generation and propagation of $\mathrm{G}$ waves from the Niiagata Earthquake of June 16, 1964. Part 2. Estimation of Earthquake Moment, Released Energy and Stress-strain Drop from the $\mathrm{G}$ wave Spectrum: Bull. Earth. Res. Ins, 44, 73-88.

Anderson J.G., Singh S.K., Espindola J.M., Yamamoto J., 1989, Seismic strain release in the mexican subduction thrust, Phys. Earth Planet. Inter., 58, 307-322.

Burbach, G.V., Frohlich C., Pennington W.D., Matumoto T., 1984, Seismicity and Tectonics of the subducted Cocos plate: J. Geophys. Res., 89, B9, 7719-7735.

Cárdenas, Monroy C., 2003, Estructura tridimensional de la zona de subducción en Guerrero, México: Un análisis de trazado de rayos con base en sismos regionales mexicanos.: D.F., México, UNAM, Posgrado en Ciencias de la Tierra. Tesis de maestría.

DeMets, C., Gordon, R., Argus, D., Stein, S., 1994, Effect of recent revisions to the geomagnetic time-scale on estimate of current plate motions. Geophys. Res. Lett., 21, 2191-2194.

Figueroa, J., 1974, Isosistas de macrosismos mexicanos: D.F., México, Instituo de Ingeniería, UNAM, 33, 1, 45-67.

Franco S.I., Iglesias A., Pacheco J.F., Singh S.K., Fukuyama E., Pérez Santana J., y Yi T., 2002, Inversión automática del tensor de momentos utilizando datos de la red de banda ancha del SSN (resumen), en Tercera Reunión anual de la Unión Geofísica Mexicana:, Pto. Vallarta, Jal., Mex., GEOS, 379-380.

Franco, S.I., 2006, Redes GPS y su aplicación en sismotectónica: D.F., México, UNAM, Posgrado en Ciencias de la Tierra. Tesis de Doctorado.

García D., S. K. Singh, M. Ordaz, J. F. Pacheco, 2005, Inslab Earthquakes of Central Mexico: Peak Ground-Motion Parameters and Response Spectra: Bull. Seism. Soc. Am., 95, 6, 2272-2282, doi: $10.1785 / 0120050072$.

Kostoglodov, V., Pacheco, J., 1999, Un catálogo de sismos moderados y grandes ocurridos en México durante el siglo XX, Póster "100 años de sismicidad en México": D.F., México, Instituto de Geofísica, UNAM.

Nolasco-Carteño L. Z., 2006, Un sistema Automático para el cálculo del tensor de momentos para sismos mexicanos: D.F., México, UNAM, Facultad de Ingeniería. Tesis de Licenciatura.

Pardo, M., Suárez, G., 1995, Shape of the subducted Rivera and Cocos plates in southern Mexico: Seismic and tectonic implications: $J$. Geophys. Res., 100, 12357-12373.

Singh, S.K., Astiz, L., Havskov, J., 1981, Seismic gaps and recurrence periods of large earthquakes along the Mexican subduction zone: a reexamination: Bull. Seism. Soc. Am., 71, 827-843.

Singh S.K. y J. F. Pacheco, 1994, Magnitude determination of Mexican earthquakes: Geofísica Internacional, 33, 2, 189-198.

Suárez, G., T. Monfret, G. Wittlinger, and C. David, 1990, Geometry of subduction and depth of seismogenic zone in the Guerrero gap, Mexico: Nature, 345, 336-338.

Tan Y. y Jiménez-Cruz C., 2002, Zonificación de Intensidades de los Sismos Grandes Por Internet (resumen), en Tercera Reunión anual de la Unión Geofísica Mexicana:, Pto. Vallarta, Jal., Mex., GEOS, 372.

Manuscrito recibido: Mayo 2, 2007

Manuscrito corregido recibido: Junio 15, 2007

Manuscrito aceptado: Julio 3, 2007 